\title{
Energy Conditions in $f(G)$ Modified Gravity with Non-minimal Coupling to Matter
}

\author{
A. Banijamali ${ }^{a}$ 1, $\quad$ B. Fazlpour ${ }^{b} 2$ and M. R. Setare S $^{c} \sqrt{3}$ \\ a Department of Basic Sciences, Babol University of Technology, Babol, Iran \\ ${ }^{b}$ Babol Branch, Islamic Azad University, Babol, Iran \\ ${ }^{c}$ Department of Science, Campus of Bijar, University of Kurdistan \\ Bijar, IRAN.
}

\begin{abstract}
In this paper we study a model of modified gravity with non-minimal coupling between a general function of the Gauss-Bonnet invariant, $f(G)$, and matter Lagrangian from the point of view of the energy conditions. Such model has been introduced in Ref. [21] for description of early inflation and late-time cosmic acceleration. We present the suitable energy conditions for the above mentioned model and then, we use the estimated values of the Hubble, deceleration and jerk parameters to apply the obtained energy conditions to the specific class of modified Gauss-Bonnet models.
\end{abstract}

PACS numbers: 04.50.-h, 04.50.kd, 98.80.-k

Keywords: Modified Gauss-Bonnet gravity, Non-minimal coupling, Energy conditions

\footnotetext{
${ }^{1}$ a.banijamali@nit.ac.ir

2b.fazlpour@umz.ac.ir

${ }^{3}$ rezakord@ipm.ir
} 


\section{Introduction}

Nowadays it is strongly believed that the universe is experiencing an accelerated expansion, and this is supported by many cosmological observations [1-4]. This accelerated expansion can be explained in terms of the so called dark energy ( for reviews see [5]) in the framework of general relativity or by modification of general relativity.

The simplest type of modified gravity models is well known as $f(R)$ gravity where the Ricci scalar in the Einstein-Hilbert action is replaced by a general function of the scalar curvature (see $[6,7]$ for reviews).

There are also other modified gravity models which are the generalization of $f(R)$ gravity and among them, the modified Gauss-Bonnet (GB) gravity i.e. $f(G)$ gravity, is more interesting $[8,9]$. The GB combination, $G$, is a topological invariant in four dimensions, so in order to play some roles in the field equations, one needs either couple GB term to a scalar field like $f(\phi) G$, or choose $f(G)$ gravity where $f$ is an arbitrary function of $G$.

If we compare $f(G)$ gravity with other theories of modified gravity we find some advantages in the Gauss-Bonnet gravity. For example in the context of $f(G)$ gravity there exists a de-Sitter point that can be used for cosmic acceleration $[8,9,21]$. Note that in $f(G)$ gravity, there are no problems $[8,9]$ with the Newton law, instabilities and the anti-gravity regime. In comparing with the simple $f(R)$ modified gravity we should mentioned that it is not generally easy to construct viable $f(R)$ models that are consistent with cosmological and local gravity constraints. The main reason for this is that $f(R)$ gravity gives rise to a strong coupling between DE and a non-relativistic matter in the Einstein frame [37]. However there is no conformal transformation separating $G$ from scalar field, unlike the $f(R)$ theory to obtain and Einstein frame action with a canonical scalar field coupled to matter. Furthermore the $f(G)$ models might be less constrained by local gravity constraints relative to the $f(R)$ models. The main reason is that even in the vacuum spherically symmetric background the Gauss-Bonnet scalar takes a non-vanishing value. This property is different from $f(R)$ gravity in which the Ricci scalar $R$ vanishes in the vacuum spherically symmetric background [38]. Moreover, In considering alternative higher-order gravity theories, one is liable to be motivated in pursuing models consistent and inspired by several candidates of a fundamental theory of quantum gravity. Indeed, motivations from string/M- theory predict that scalar field couplings with the Gauss- Bonnet invariant, $G$, are important in the appearance of non-singular early time cosmologies [33]. In summary, modified $f(G)$ gravity represents a quite interesting gravitational alternative to dark energy with more freedom if compare with $f(R)$ gravity (for recent review see [14]).

The $f(G)$ gravity is an enrichment theory of modified gravity. A number of its abilities are as follows: it has the possibility to describe the inflationary era, a transition from a deceleration phase to an acceleration phase, crossing the phantom divide line and passing the solar system tests for a reasonable defined function $f$ [10-13].

Moreover, a gravitational source of the inflation and dark energy may be the non-minimal coupling of some geometrical invariants function with matter Lagrangian. Such non-minimal modified gravity has been introduced in Refs. $[15,16]$ for the study of gravity assisted dark energy occurrence. It may be also applied for realization of dynamical cancellation of cos- 
mological constant [17]. The viability criteria for such theory was recently discussed in Refs. [18-20]. Non- minimal coupling of $f(G)$ modified gravity with matter Lagrangian has been investigated in [21]. It is shown [21], that such a model can easily unify the early inflation with late-time cosmic acceleration for the special choise of gravitational functions. Clearly, as any model in $f(G)$ theory, there are particular conditions which have to be satisfied in order to ensure that the model is viable and physically meaningful [22-24]. In the present work we study the above mentioned model of $f(G)$ gravity with non-minimal coupling to matter from the viewpoint of the energy conditions.

Furthermore, the energy conditions are essential for studying the singularity theorems as well as the theorems of black hole thermodynamics. For example the Hawking-Penrose singularity theorems invoke the weak and strong energy conditions, whereas the proof of the second law of black hole thermodynamics needs the null energy condition [25]. Various forms of energy conditions namely, strong, weak, dominant and null energy conditions are obtained using Raychaudhuri equation along with attractiveness property of gravity [25, 26]. Energy conditions have been widely studied in the literature. Phantom field potential [27], expansion history of the universe [28] and evolution of the deceleration parameter [29] have been investigated using classical energy conditions of general relativity. Energy conditions in the context of $f(R)$ gravity have been derived in [30] and following the formalism developed in [30], several authors have studied some cosmological issues in modified $f(R)$ gravity from this point of view (see for example [31, 32]). Also, appropriate energy conditions for the $f(R)$ gravity non-minimally coupled with matter Lagrangian has been investigated in [22]. In a recent paper [33], the general energy conditions for modified Gauss-Bonnet gravity or $f(G)$ gravity have been presented and viability of specific realistic forms of $f(G)$ proposed in [21] have been analyzed by imposing the weak energy condition.

In this paper we generalize procedure developed in [33] for $f(G)$ theories to the gravity model with non-minimal coupling of $f(G)$ to the matter. Then, we use the estimated values of the Hubble, deceleration and jerk parameters to apply the obtained energy conditions to the specific class of models.

An outline of this paper is as follows: in the next section we review the field equations of modified $f(G)$ theory non-minimally coupled with matter. In section 3 we present the suitable energy conditions for such a model. In section 4 we examine the weak energy condition for two specific class of $f(G)$ models. Section 5 is devoted to our conclusions.

\section{Field Equations}

Our starting action for modified Gauss-Bonnet gravity non-minimally coupled with matter is as follows [21]:

$$
S=\int d^{4} x \sqrt{-g}\left[\frac{1}{2 \kappa^{2}} R+f(G) \mathcal{L}_{m}\right]
$$

where $g$ is the determinant of the metric tensor $g_{\mu \nu}, R$ is the Ricci scalar and $f(G)$ is a general function of Gauss-Bonnet invariant $G$. One notes that there is a non-minimal 
coupling between $f(G)$ gravity and the matter Lagrangian $\mathcal{L}_{m}$ in (1).

The theory of kind (1) has been previously investigated in order to unify inflation and latetime cosmic acceleration [21].

The Gauss-Bonnet term is given by,

$$
G=R^{2}-4 R_{\mu \nu} R^{\mu \nu}+R_{\mu \nu \rho \sigma} R^{\mu \nu \rho \sigma} .
$$

Varying the action (1) with respect to $g_{\mu \nu}$ leads to:

$$
\begin{aligned}
0 & =\frac{1}{2 \kappa^{2}}\left(R_{\mu \nu}-\frac{1}{2} g_{\mu \nu} R\right)+2 f^{\prime}(G) \mathcal{L}_{m} R R_{\mu \nu} \\
& -4 f^{\prime}(G) \mathcal{L}_{m} R_{\mu}{ }^{\rho} R_{\nu \rho}+2 f^{\prime}(G) \mathcal{L}_{m} R_{\mu \rho \sigma \lambda} R_{\nu}{ }^{\rho \sigma \lambda}+4 f^{\prime}(G) \mathcal{L}_{m} R_{\mu \rho \sigma \nu} R^{\rho \sigma} \\
& +2\left(\left(g_{\mu \nu} \square-\nabla_{\mu} \nabla_{\nu}\right) f^{\prime}(G) \mathcal{L}_{m}\right) R+4\left(\nabla^{\rho} \nabla_{\mu} f^{\prime}(G) \mathcal{L}_{m}\right) R_{\nu \rho}+4\left(\nabla^{\rho} \nabla_{\nu} f^{\prime}(G) \mathcal{L}_{m}\right) R_{\mu \rho} \\
& +4\left(\square f^{\prime}(G) \mathcal{L}_{m}\right) R_{\mu \nu}-4\left(g_{\mu \nu} \nabla^{\lambda} \nabla^{\rho} f^{\prime}(G) \mathcal{L}_{m}\right) R_{\lambda \rho}+4\left(\nabla^{\rho} \nabla^{\lambda} f^{\prime}(G) \mathcal{L}_{m}\right) R_{\mu \rho \nu \lambda}+f(G) T_{\mu \nu}
\end{aligned}
$$

where,

$$
T_{\mu \nu}=\frac{2}{\sqrt{-g}} \frac{\delta}{\delta g^{\mu \nu}}\left(\int d^{4} x \sqrt{-g} \mathcal{L}_{m}\right)
$$

is the energy-momentum tensor of the ordinary matter and prime stands for derivative with respect to $G$. For a flat Friedman-Robertson-Walker (FRW) metric with scale factor $a(t)$,

$$
d s^{2}=-d t^{2}+a^{2}(t)\left(d r^{2}+r^{2} d \Omega^{2}\right),
$$

and assuming matter as a perfect fluid, the field equation (3) has the following simple forms,

$$
\begin{aligned}
& \frac{3}{\kappa^{2}} H^{2}+24 H^{3} \frac{d}{d t}\left(f^{\prime}(G) \mathcal{L}_{m}\right)-G f^{\prime}(G) \mathcal{L}_{m}=f(G) \rho \\
& \frac{1}{\kappa^{2}}\left(2 \dot{H}+3 H^{2}\right)+8 H^{2} \frac{d^{2}}{d t^{2}}\left(f^{\prime}(G) \mathcal{L}_{m}\right)+16 H \frac{d}{d t}\left(f^{\prime}(G) \mathcal{L}_{m}\right)\left(\dot{H}+H^{2}\right)-G f^{\prime}(G) \mathcal{L}_{m}=-f(G) p
\end{aligned}
$$

where $\rho$ and $p$ are the energy density and pressure, respectively. The overdot denotes a time derivative.

In order to write the field equations as those in general relativity, one can define the effective energy density and pressure,

$$
\rho_{\text {eff }}=\frac{3}{\kappa^{2}} H^{2}
$$

and

$$
p_{e f f}=-\frac{1}{\kappa^{2}}\left(2 \dot{H}+3 H^{2}\right),
$$

where $\rho_{\text {eff }}$ and $p_{\text {eff }}$ are the effective energy density and pressure, respectively and given by,

$$
\rho_{e f f}=f(G)\left[G \frac{f^{\prime}(G)}{f(G)} \mathcal{L}_{m}-24 H^{3} \dot{G} \frac{f^{\prime \prime}(G)}{f(G)} \mathcal{L}_{m}+\rho\right]
$$


and

$p_{\text {eff }}=f(G)\left[-G \frac{f^{\prime}(G)}{f(G)} \mathcal{L}_{m}+8 H^{2}\left(\ddot{G} \frac{f^{\prime \prime}(G)}{f(G)}+\dot{G}^{2} \frac{f^{\prime \prime \prime}(G)}{f(G)}\right) \mathcal{L}_{m}+16 H \dot{G} \frac{f^{\prime \prime}(G)}{f(G)}\left(\dot{H}+H^{2}\right) \mathcal{L}_{m}+p\right]$,

where, we have used the equations (6) and (7).

\section{Energy conditions}

The energy conditions originate from the Raychaudhury equation together with the attractiveness of the gravity [26]. The Raychaudhury equation in the case of a congruence of null geodesics defined by the vector field $k^{\mu}$ is given by,

$$
\frac{d \theta}{d \tau}=-\frac{1}{2} \theta^{2}-\sigma_{\mu \nu} \sigma^{\mu \nu}+\omega_{\mu \nu} \omega^{\mu \nu}-R_{\mu \nu} k^{\mu} k^{\nu},
$$

where $R_{\mu \nu}$ is the Ricci tensor, $\theta$ is the expansion parameter, $\sigma_{\mu \nu}$ and $\omega_{\mu \nu}$ are the shear and the rotation associate to the congruence respectively.

For any hypersurface of orthogonal congruences $\left(\omega_{\mu \nu}=0\right)$, the condition of attractiveness of gravity $\left(\frac{d \theta}{d \tau}<0\right)$ yields to $R_{\mu \nu} k^{\mu} k^{\nu} \geq 0\left(\sigma_{\mu \nu} \sigma^{\mu \nu} \geq 0\right)$.

This condition in the context of general relativity expresses by $T_{\mu \nu} k^{\mu} k^{\nu} \geq 0$.

The Raychaudhury equation satisfies for any geometrical theory of gravitation. In the other hand the theory of kind (1) has an Einstein-Hilbert term and evaluating $R_{\mu \nu} k^{\mu} k^{\nu}$ is straightforward. So, using the effective gravitation field equations, the energy conditions in the case of our model are given by,

$$
\begin{gathered}
\rho_{e f f}+p_{e f f} \geq 0 \quad \text { for null energy condition }(N E C), \\
\rho_{e f f} \geq 0 \text { and } \rho_{e f f}+p_{e f f} \geq 0 \quad \text { for weak energy condition }(W E C), \\
\rho_{\text {eff }}+3 p_{\text {eff }} \geq 0 \text { and } \rho_{\text {eff }}+p_{\text {eff }} \geq 0 \quad \text { for strong energy condition }(S E C),
\end{gathered}
$$

and

$$
\rho_{e f f} \geq 0 \text { and } \rho_{e f f} \pm p_{e f f} \geq 0 \quad \text { for dominant energy condition }(D E C) .
$$

Inserting equations (10) and (11) into equations (13)-(16) lead to the following respective forms,

$$
\begin{aligned}
N E C: \rho+p-8 & H^{3} \dot{G} \frac{f^{\prime \prime}(G)}{f(G)} \mathcal{L}_{m}+8 H^{2}\left(\ddot{G} \frac{f^{\prime \prime}(G)}{f(G)}+\dot{G}^{2} \frac{f^{\prime \prime \prime}(G)}{f(G)}\right) \mathcal{L}_{m} \\
+ & 16 H \dot{H} \dot{G} \frac{f^{\prime \prime}(G)}{f(G)} \mathcal{L}_{m} \geq 0
\end{aligned}
$$




$$
\begin{gathered}
W E C: \rho+G \frac{f^{\prime}(G)}{f(G)} \mathcal{L}_{m}-24 H^{3} \dot{G} \frac{f^{\prime \prime}(G)}{f(G)} \mathcal{L}_{m} \geq 0, \quad \rho_{\text {eff }}+p_{\text {eff }} \geq 0 \\
S E C: \rho+3 p-2 G \frac{f^{\prime}(G)}{f(G)} \mathcal{L}_{m}+24 H^{3} \dot{G} \frac{f^{\prime \prime}(G)}{f(G)} \mathcal{L}_{m}+24 H^{2}\left(\ddot{G} \frac{f^{\prime \prime}(G)}{f(G)}+\dot{G}^{2} \frac{f^{\prime \prime \prime}(G)}{f(G)}\right) \mathcal{L}_{m} \\
+48 H \dot{H} \dot{G} \frac{f^{\prime \prime}(G)}{f(G)} \mathcal{L}_{m} \geq 0, \quad \rho_{e f f}+p_{e f f} \geq 0 . \\
D E C: \rho-p+2 G \frac{f^{\prime}(G)}{f(G)} \mathcal{L}_{m}-40 H^{3} \dot{G} \frac{f^{\prime \prime}(G)}{f(G)} \mathcal{L}_{m}-8 H^{2}\left(\ddot{G} \frac{f^{\prime \prime}(G)}{f(G)}+\dot{G}^{2} \frac{f^{\prime \prime \prime}(G)}{f(G)}\right) \mathcal{L}_{m} \\
-16 H \dot{H} \dot{G} \frac{f^{\prime \prime}(G)}{f(G)} \mathcal{L}_{m} \geq 0, \quad \rho_{e f f}+p_{\text {eff }} \geq 0, \quad \rho_{e f f} \geq 0 .
\end{gathered}
$$

In order to analyze the model of type (1) with non-minimal coupling between $f(G)$ gravity and matter from the point of view of energy conditions, we use the standard terminology in studying energy conditions for modified gravity theories. To this end, according to the Hubble parameter $H=\frac{\dot{a}}{a}$ we define the deceleration $(q)$, jerk $(j)$, and snap $(s)$ parameters as,

$$
q=-\frac{1}{H^{2}} \frac{\ddot{a}}{a}, \quad j=\frac{1}{H^{3}} \frac{\dddot{a}}{a}, \quad \text { and } \quad s=\frac{1}{H^{4}} \frac{\dddot{a}}{a} .
$$

In terms of above parameters, time derivatives of the Hubble parameter can be expressed as,

$$
\begin{gathered}
\dot{H}=-H^{2}(1+q), \\
\ddot{H}=H^{3}(j+3 q+2), \\
\dddot{H}=H^{4}(s-2 j-5 q-3) .
\end{gathered}
$$

The Gauss-Bonnet invariant in FRW background is as $G=24 H^{2}\left(H^{2}+\dot{H}\right)$. So, $G$ and its time derivatives can be written as,

$$
\begin{gathered}
G=-24 H^{4} q, \\
\dot{G}=24 H^{5}\left(2 q^{2}+3 q+j\right), \\
\ddot{G}=-24 H^{6}\left(2 q^{3}+22 q^{2}+6 q j+15 q+4 j-s-3\right) .
\end{gathered}
$$

By inserting relations (22)-(27) into equations (17)-(20) one can obtain the following forms of energy conditions

$$
\begin{gathered}
\rho_{0}+p_{0}-192 H_{0}^{8}\left(5 q_{0}^{3}+34 q_{0}^{2}+8 q_{0} j_{0}+24 q_{0}+7 j_{0}-s_{0}-3\right) \frac{f_{0}^{\prime \prime}(G)}{f_{0}(G)} \mathcal{L}_{m} \\
+4608 H_{0}^{12}\left(2 q_{0}^{2}+3 q_{0}+j_{0}\right)^{2} \frac{f_{0}^{\prime \prime \prime}(G)}{f_{0}(G)} \mathcal{L}_{m} \geq 0
\end{gathered}
$$


for null,

$$
\rho_{0}-24 H_{0}^{4} q_{0} \frac{f_{0}^{\prime}(G)}{f_{0}(G)} \mathcal{L}_{m}-576 H_{0}^{8}\left(2 q_{0}^{2}+3 q_{0}+j_{0}\right) \frac{f_{0}^{\prime \prime}(G)}{f_{0}(G)} \mathcal{L}_{m} \geq 0,
$$

for weak,

$$
\begin{gathered}
\rho_{0}+3 p_{0}+48 H_{0}^{4} q_{0} \frac{f_{0}^{\prime}(G)}{f_{0}(G)} \mathcal{L}_{m}-576 H_{0}^{8}\left(6 q_{0}^{3}+30 q_{0}^{2}+8 q_{0} j_{0}+18 q_{0}+5 j_{0}-s_{0}-3\right) \frac{f_{0}^{\prime \prime}(G)}{f_{0}(G)} \mathcal{L}_{m} \\
+13824 H_{0}^{12}\left(2 q_{0}^{2}+3 q_{0}+j_{0}\right)^{2} \frac{f_{0}^{\prime \prime \prime}(G)}{f_{0}(G)} \mathcal{L}_{m} \geq 0
\end{gathered}
$$

for strong and

$$
\begin{gathered}
\rho_{0}-p_{0}-48 H_{0}^{4} q_{0} \frac{f_{0}^{\prime}(G)}{f_{0}(G)} \mathcal{L}_{m}+192 H_{0}^{8}\left(6 q_{0}^{3}+22 q_{0}^{2}+8 q_{0} j_{0}+6 q_{0}+j_{0}-s_{0}-3\right) \frac{f_{0}^{\prime \prime}(G)}{f_{0}(G)} \mathcal{L}_{m} \\
-4608 H_{0}^{12}\left(2 q_{0}^{2}+3 q_{0}+j_{0}\right)^{2} \frac{f_{0}^{\prime \prime \prime}(G)}{f_{0}(G)} \mathcal{L}_{m} \geq 0
\end{gathered}
$$

for dominant energy condition respectively. The subscript 0 stands for the present value of quantities. These forms of energy conditions are suitable to impose bounds on a given $f(G)$ model by using the estimate values of the $q_{0}, j_{0}$ and $s_{0}$.

\section{Specific models}

Now, let us see how the above energy conditions work for specific type of $f(G)$ models. In what follows we focus just on WEC inequality (29). Our reason apart from the simplicity is that all other energy conditions depend on the present value of snap parameter $s_{0}$ and as mentioned in [30] until now no reliable measurement of this parameter has been reported. Also, for simplicity we only examine the case in which $p=\rho=0$, although this is not a physically interesting case, but this is easily corrected, since one can always add a positive energy density or pressure from matter and/or radiation to any model satisfying the WEC , and it will still satisfy the WEC [33].

Case 1: Our first example is coming from Ref. [9],

$$
f(G)=\alpha G^{n} .
$$

It has been shown in [9] that this type of $f(G)$ theory can produce quintessence, phantom or cosmological constant cosmology and has the possibility of realizing transition from the deceleration to the acceleration era. It was shown in [39] that the model of type (32) with $n<0$, is not cosmologically viable because of separatrices between radiation and dark energy dominations. In [38] it was found that the model (32) with $n>0$, can be consistent with 
solar system tests for $n \leq 0.074$ if the Gauss-Bonnet term is responsible for dark energy. Inserting $f(G)$ from (32) and their derivatives into equation (29) for WEC yields to

$$
(-1)^{n} \alpha\left[A n^{2}-(A+1) n\right] \mathcal{L}_{m} \leq 0
$$

where $A=\frac{2 q^{2}+3 q+j}{q^{2}}$.

The following estimated values of deceleration and jerk parameters $[34,35] ; q_{0}=-8.81 \pm 0.14$ and $j_{0}=2.16_{-.75}^{+0.81}$ allow us to further analyze equation (33). Note that the roots of $\left[A n^{2}-(A+1) n\right]=0$ are $\left(0,1+\frac{1}{A}\right)$, so it has positive values for $0>n>1.63$ and negative values for $0 \leq n \leq 1.63$. In addition, the condition required for attractiveness of gravity i.e. $f(G)>0$ leads to $\alpha(-1)^{n}(2.09 H)^{4 n}>0$. Now, using the above explanations, one can study the inequality (33) as follows:

(i) for $\alpha>0$, one concludes from the attractivness property of gravity that $n$ should be even and then the equation (33) tell us that $0 \leq n \leq 1.63$, and therefore there is no allowed value for $n$.

(ii) for $\alpha<0, n$ should be odd and equation (33) leads to $0>n>1.63$ so the allowed sets of $n$ s are $n=\{3,5,7, \ldots\}$ and $n=\{-1,-3,-5, \ldots\}$.

Case 2: In the second example we consider a class of models as follows:

$$
f(G)=\alpha G^{n}\left(1+\beta G^{m}\right) .
$$

The models of kind (34) have been proposed in Ref. [21] in order to address the late-time cosmic acceleration. It has been shown in [40] that the model (34) is consistent with local tests and cosmological bounds. The typical property of such theory is the presence of the effective cosmological constant epochs in such a way that early-time inflation and late-time cosmic acceleration are naturally unified within single model. It is shown that classical instability does not appear here and Newton law is respected. Some discussion of possible anti-gravity regime appearance and related modification of the theory is also done in [40]. Furthermore, it is shown in [36] that four types of future singularities can be cured in such a models if one considers $n>0$ and $m<0$.

By inserting the above $f(G)$ and their derivatives into equation (29), WEC reads

$$
\left[\alpha(-1)^{n} a_{n}+\alpha \beta(-1)^{n+m}\left(a_{n}+a_{m}+2 A m n\right)\left(24 H^{4} q\right)^{m}\right] \mathcal{L}_{m} \leq 0,
$$

where $a_{n}=A n^{2}-(A+1) n$ and $a_{n}+a_{m}+2 A m n=A(n+m)^{2}-(A+1)(n+m)$. For the next purposes, we mention the roots of $a_{n}=0$ and $a_{n}+a_{m}+2 A m n=0$ are $n=\left(0,1+\frac{1}{A}\right)$ and $n+m=\left(0,1+\frac{1}{A}\right)$ respectively. So, they have positive values for $0>n>1.63$ and $0>n+m>1.63$ and negative values for $0 \leq n \leq 1.63$ and $0 \leq n+m \leq 1.63$ respectively. In addition, the condition $f(G)>0$ leads to $\alpha(-1)^{n}(2.09 H)^{4 n}\left(1+\beta(-1)^{m}(2.09 H)^{4 m}\right)>0$. Now, by using the above necessary condition one can study the inequality (35) as the following cases: 
(i) In the first situation we assume that $a_{n}>0$ and $a_{n}+a_{m}+2 A m n>0$ or $a_{n}<0$ and $a_{n}+a_{m}+2 A m n<0$ so we have the following possibilities:

(i1) for $\alpha(-1)^{n}>0$, if $\left|\frac{a_{n}}{a_{n}+a_{m}+2 A m n}\right|(2.09 H)^{-4 m} \leq \beta<(2.09 H)^{-4 m}$ then $m$ should be odd and if $-(2.09 H)^{-4 m}<\beta<-\left|\frac{a_{n}}{a_{n}+a_{m}+2 A m n}\right|(2.09 H)^{-4 m}$ then $m$ should be even.

(i2) for $\alpha(-1)^{n}<0$, it is impossible to realize the conditions (35) and $f(G)>0$ simultaneously.

(ii) Here, in the second situation we present the conditions required for WEC fulfilment for $a_{n}<0$ and $a_{n}+a_{m}+2 A m n>0$ or $a_{n}>0$ and $a_{n}+a_{m}+2 A m n<0$ :

(ii1) for $\alpha(-1)^{n}>0$, if $\beta \geq\left|\frac{a_{n}}{a_{n}+a_{m}+2 A m n}\right|(2.09 H)^{-4 m}$ then $m$ should be even and if $\beta \leq-\left|\frac{a_{n}}{a_{n}+a_{m}+2 A m n}\right|(2.09 H)^{-4 m}$ then $m$ should be odd.

(ii2) for $\alpha(-1)^{n}<0$, if $\beta>(2.09 H)^{-4 m}$ then $m$ should be odd and if $\beta<-(2.09 H)^{-4 m}$ then $m$ should be even.

\section{Conclusion}

In this work we investigated a model of modified gravity with non-minimal coupling between modified Gauss-Bonnet gravity, $f(G)$, and matter Lagrangian described by action (1) from the viewpoint of the energy conditions. We derived the suitable energy conditions inequalities for such a model as equations (17)- (20).

We examined the WEC inequality equation (29) for two class of viable models of $f(G)$ gravity presented in equations (32) and (34). We concluded that the model $f(G)=\alpha G^{n}$ obey the WEC only for $\alpha<0$. For the model $f(G)=\alpha G^{n}\left(1+\beta G^{m}\right)$ our result summarized in subcases (i1), (i2), (ii1) and (ii2). One can use our method and in particular equations (28)-(31) to study the physical implications of any $f(G)$ model with non-minimal coupling to matter.

Finally, we mention the following important point: as emphasized in [30], although the energy conditions in modified gravity theories have well-founded physical motivation (the Raychaudhury equation together with the attractiveness property of gravity) the question as to whether they should be applied to any modified gravity theory is an open question which is ultimately related to the confrontation between theory and observations.

\section{References}

[1] S. Perlmutter, et al., Supernova Cosmology Project Collaboration, Astrophys. J. 517, 565 (1999). 
[2] C. L. Bennett, et al., Astrophys. J. Suppl. 148, 1 (2003).

[3] M. Tegmark, et al., SDSS Collaboration, Phys. Rev. D 69, 103501 (2004).

[4] S.W. Allen, et al., Mon. Not. R. Astron. Soc. 353, 457 (2004).

[5] E. J. Copeland, M. Sami, S. Tsujikawa, Int. J. Mod. Phys. D 15, 1753 (2006); Y. F. Cai, E. N. Saridakis, M. R. Setare, J. Q. Xia, Physics Reports 493, 1 (2010); M. Li, X. D. Li, S. Wang, Y. Wang, arXiv:1103.5870 [astro-ph.CO].

[6] S. Nojiri and S. D. Odintsov, Int. J. Geom. Math. Mod. Phys. 4, 115 (2007).

[7] S. Nojiri and S. D. Odintsov, arXiv:0801.4843 [astro-ph]; arXiv:0807.0685 [hep-th]; T. P. Sotiriou and V. Faraoni, Rev. Mod. Phys. 82, 451 (2010); F. S. N. Lobo, arXiv:0807.1640 [gr-qc] and S. Capozziello and M. Francaviglia, Gen. Rel. Grav. 40, 357 (2008).

[8] S. Nojiri and S. D. Odintsov, Phys. Lett. B 631, 1 (2005).

[9] G. Cognola, E. Elizalde, S. Nojiri, S. D. Odintsov and S. Zerbini, Phys. Rev. D 75, 086002 (2007).

[10] S. Nojiri, S. D. Odintsov, S. Ogushi, Int. J. Mod. Phys. A 17, 4809 (2002); B. M. Leith, I. P. Neupane, J. Cosmol. Astropart. Phys. 0705, 019 (2007).

[11] J. Sadeghi, M. R. Setare and A. Banijamali, Phys. Lett. B 679, 302 (2009); J. Sadeghi, M. R. Setare and A. Banijamali, Eur. Phys. J. C 64, 433 (2009); M. R. Setare and E. N. Saridakis , Phys. Lett. B 670, 1 (2008); M. R. Setare, Int. J. Mod. Phys. D 17, 2219 (2008); M. R. Setare, Chin. Phys. Lett. 26, 029501 (2009).

[12] A. De Felice, S. Tsujikawa, Phys. Lett. B 675, 1 (2009).

[13] A. De Felice, S. Tsujikawa, Phys. Rev. D 80, 063516 (2009).

[14] S. Nojiri and S. D. Odintsov, arXiv:1011.0544 [gr-qc]; S. Capozziello, M. D. Laurentis, arXiv:1108.6266r 2 [gr-qc].

[15] S. Nojiri and S. D. Odintsov, Phys. Lett. B 599, 137 (2004); PoS WC2004, 024 (2004) arXiv:0412030[hep-th].

[16] G. Allemandi, A. Borowiec, M. Francaviglia, and S. D. Odintsov, Phys. Rev. D 72, 063505 (2005).

[17] S. Mukohyama and L. Randall, Phys. Rev. Lett. 92, 211302 (2004); T. Inagaki, S. Nojiri and S. D. Odintsov, JCAP 0506, 010 (2005) arXiv:gr-qc/0504054; A. D. Dolgov and M. Kawasaki, arXiv:0307442[astro-ph].

[18] O. Bertolami, C. Boehmer, T. Harko, and F. Lobo, Phys. Rev. D 75, 104016 (2007); T. Koivisto, Class. Quant. Grav. 23, 4289 (2006). 
[19] V. Faraoni, Phys. Rev. D 76, 127501 (2007).

[20] O. Bertolami and J. Paramos, Phys. Rev. D 77, 084018 (2008).

[21] S. Nojiri, S. D. Odintsov, and P. Tretyakov, Phys. Lett. B 651, 224 (2007); S. Nojiri, S. D. Odintsov and P. V. Tretyakov, Prog. Theor. Phys. Supp 172, 81 (2008).

[22] O. Bertolami and M. C. Sequeira, Phys. Rev. D 79, 104010 (2009).

[23] J. H. Kung, Phys. Rev. D 52, 6922 (1995) ; Phys. Rev. D 53, 3017 (1996).

[24] S. E. Perez Bergliaffa, Phys. Lett. B 642, 311 (2006).

[25] S. W. Hawking and G. F. R. Ellis, The Large Scale Structure of Spacetime (Cambridge University Press, England, 1973); R. M. Wald, General Relativity (University of Chicago Press, Chicago, 1984).

[26] S. Carroll, Spacetime and Geometry: An Introduction to General Relativity, (Addison Wesley, New York, 2004).

[27] J. Santos and J. S. Alcaniz, Phys. Lett. B 619, 11 (2005).

[28] M. Visser, Science 276, 88 (1997); Phys. Rev. D 56, 7578 (1997) ; J. Santos, J. S. Alcaniz and M. J. Reboucas, Phys. Rev. D 74, 067301 (2006); J. Santos, J. S. Alcaniz, N. Pires and M. J. Reboucas, Phys. Rev. D 75, 083523 (2007); A. A. Sen and R. J. Scherrer, Phys. Lett. B 659, 457 (2008); J. Santos, J. S. Alcaniz, M. J. Reboucas and N. Pires, Phys. Rev. D 76, 043519 (2007).

[29] Y. G. Gong, A. Wang, Q. Wu and Y. Z. Zhang, JCAP 0708, 018 (2007); Y. Gong and A. Wang, arXiv:0705.0996v1 [astro-ph].

[30] J. Santos, J. S. Alcaniz, M. J. Reboucas and F. C. Carvalho, Phys. Rev. D 76, 083513 (2007).

[31] J. Santos, M. J. Reboucas and J. S. Alcaniz, Int. J. Mod. Phys. D 19, 1315 (2010).

[32] K. Atazadeh, A. Khaleghi, H. R. Sepangi and Y. Tavakoli, Int. J. Mod. Phys. D 18, 1101 (2009).

[33] N. M. Garcia, T. Harko, Francisco S. N. Lobo and Jose P. Mimoso, Phys. Rev. D 83, 104032 (2011).

[34] D. Rapetti, S. W. Allen, M. A. Amin and R.D. Blandford, Mont. Not. R. Soc. 375, 1510 (2007).

[35] N. J. Poplawski, Class. Quant. Grav. 24, 3013 (2007).

[36] K. Bamba, S. D. Odintsov, L. Sebastiani and S. Zerbini, Eur. Phys. J. C 67, 295 (2010). 
[37] L. Amendola, D. Polarski and S. Tsujikawa,Phys. Rev. Lett 98, 131302 (2007).

[38] S. C. Davis, arXiv:0709.4453 [hep-th].

[39] A. De Felice and M. Hindmarsh, JCAP 0706, 028 (2007).

[40] S. Nojiri and S. D. Odintsov,Phys. Lett. B 657, 238 (2007). 